\title{
Inner-shell ionization cross section of gold by elec- tron and positron impact
}

\author{
Yogesh $\operatorname{Kumar}^{a *}$ and Monoj Kumar ${ }^{b}$ \\ a Department of Physics, D.A.V College, Muzaffernagar 251001, India \\ ${ }^{b}$ Department of Physics, Meerut College, Meerut 250001, India
}

Received 25 June 2014; Accepted (in revised version) 4 September 2014

Published Online 29 October 2014

\begin{abstract}
The theoretical modified Khare (BEB) model has been used to calculate the total cross sections for $\mathrm{L}_{1}, \mathrm{~L}_{2}$ and $\mathrm{L}_{3}$-subshells ionization of gold atom due to electron and positron impact for projectile energy varying from the threshold of ionization to 60 times the threshold energy For L subshells the present cross section due to electron and positron impact cross sections are in remarkable agreement with available experimental data and other theoretical cross sections. Total L shell ionization cross sections have been also calculated in the energy varying from the threshold of ionization to several $\mathrm{MeV}$ by electron impact. It is found good agreement with available experimental data. The investigation for other atoms is in progress.
\end{abstract}

PACS: $34.80 \mathrm{Dp}$

Key words: atomic ionization, electron impact, positron impact

\section{Introduction}

Electron and positron impact ionization cross sections for inner shells are needed in many areas of research such as fusion physics, plasma physics, space physics environmental protection, material analysis by various techniques etc. [1,2]. The ionization cross sections find important application in field such as atmosphere physics, radiation science, astrophysics, etc. Furthermore, the positron being an antielectron, a comparison of the electron impact ionization cross sections with those by positron impact helps to understand the basic deference between the matter-matter and matter-antimatter interactions. Over past six decades many experimental and theoretical studies have been carried out to estimate the electron and positron impact inner-shell ionization cross sections by various groups. Experimentally L shell ionization cross sections have been measured by

${ }^{*}$ Corresponding author. Email address: siwachmanoj7675@gmail . com (Y. Kumar) 
Palinka et al. [3], Middleman et al. [4], Ishii et al. [5], Shima et al. [6], Hoffmann et al. [7], Schneider et al. [8], Lennard et al. [9], Reusch et al. [10] etc. at different times by electron impact. Many theoretical researchers like Scofield [11], Khare et al. [12-14], Kim et al. [15], Haque et al. [16], etc. have calculated inner ionization cross sections due to electron impact. Scofield [11] proposed a model to calculate the ionization cross sections over wide incident energies taking into account the relativistic effect in relativistic plane wave born approximation (PWBA) through Dirac equation. His cross sections exhibit nice agreement with experimental data at ultrarelativistic energies. However, these methods fail at impact energies near threshold of ionization. Khare et al. [12-14] have calculated the electron impact ionization cross sections for Inner-shell for a numbers of atoms. They have employed the Plane Wave Born Approximation (PWBA) with corrections for exchange, coulomb and relativistic effects. Along with the longitudinal interaction, the contribution of transverse interaction to the ionization cross section is also included. Recently many researchers like Haque et al. [16] etc. have calculated the inner shell ionization cross sections by modifying the different model from threshold to ultra relativistic range.

A positron, due to its positive charge, is accelerated while passing through the atomic field, where an electron is accelerated. Furthermore, because of the distinguishability of a positron from an electron, exchange scattering does occur in positron-atom scattering. These differences have led to a number of investigations in which the ratio of the inner shell ionization cross sections of atoms, by electron and positron impacts at the same impact energy, are measured [8,9]. For positron impact, only limited researchers $[8,9]$ have carried out the inner ionization cross sections of atoms. Schneider et al. [8] have the measured the absolute $\mathrm{L}_{3}$-subshell ionization cross sections of gold by positron and electron impacts and found that at low impact energies the positron impact cross sections are much lower than those due to electron impact. Lennard et al. [9] have also obtained the ratio of L3-subshell ionization cross sections $\sigma(e-) / \sigma(e+)$ and found less than one in the energy range. Theoretically Khare et al. [12-14] have calculated the total ionization cross sections due to positron impacts for L shell for a numbers of atoms. They found the ratio of the electron-impact ionization cross section $\sigma(e-)$ to the positron- impact ionization cross section $\sigma(e+)$ is quite close to unity at high impact energies.

In 1999 Khare et al. [17] purposed model to calculate the ionization cross sections for molecules by combining the useful features of two models Kim et al. [18] and Saksena et al. [19] . In Khare (BEB) method, calculated cross sections [20-22] were in between in better agreement with the experiment data over a wide energy varying from threshold to several MeV. This model have been modified by Y. Kumar et al. [23] to obtained the K shell cross sections for many atoms $(6 \Leftarrow \mathrm{Z} \Leftarrow 92)$ in the energy varying from the threshold of ionization to $1 \mathrm{GeV}$ by electron impact. They have replaced factor $1 /\left(\mathrm{E}_{r}+\mathrm{I}+\mathrm{U}\right)$ by $1 /\left(\mathrm{E}_{r}\right.$ $+\mathrm{f})$, where $\mathrm{E}_{r}=$ relativistic energy of the incident particle, $\mathrm{I}=$ target particle's binding energy, $\mathrm{U}=$ target particle's kinetic energy and $f=\eta I_{r} /(1+\xi Z)$ is a factor which depends on atomic number (Z), here $\eta$ and $\xi$ are the experimentally fitted parameter and $I_{r}=$ target particle's binding energy with relativistic correction. The calculated cross sections were in good agreement with available experimental data. In present investigation we have 
considered that factor $\mathrm{f}$ depends angular quantum number $(l)$ and replaced $\mathrm{f}$ by $\mathrm{f} /(l+1)$ for $l$ sub-shell ionization in modified Khare (BEB) model [23] to calculate L shell ionization cross sections of gold atom due to the electron and positron impact.

\section{Theory}

In the modified Khare [BEB] model [23] for $L_{i}$ subshell the total ionization cross section is given by

$$
\sigma_{i T}=\sigma_{i P M}+\sigma_{i P B}+\sigma_{i t}
$$

With the Mott cross section

$$
\sigma_{i P M}=\left(\frac{s}{t+f}\right)\left[1-\frac{2}{t+1}+\frac{t-1}{2 t^{2}}+\frac{5-t^{2}}{(t+1)^{2}}-\frac{1}{t(t+1)}-\frac{t+1}{t^{2}}-\frac{t+1}{t^{2}} \ln \left(\frac{t+1}{2}\right)\right]
$$

Bethe cross section

$$
\sigma_{i P B}=\left(\frac{s}{t+f}\right)\left[.4431\left(1-\frac{1}{t^{2}}\right)-\frac{1}{2} \ln \left(\frac{1}{t}+\frac{l_{r}}{2 m c^{2}}\right)+\frac{1}{2 t^{2}} \ln \left(1+\frac{E_{r}}{2 m c^{2}}\right)\right]
$$

and the cross section due to transverse interaction is

$$
\sigma_{i t}=-\left(\frac{s b_{n l}}{t+f}\right)\left[\ln \left(1-\beta^{2}\right)+\beta^{2}\right]
$$

where $t=\frac{E_{r}}{I_{r}}$

Relativistic energy of projectile of mass $\mathrm{m}, E_{r}=\frac{1}{2} m c^{2}\left[1-\frac{1}{\left(1+\frac{E}{m c^{2}}\right)}\right]$ ionization energy with relativistic correction, $I_{r}=\frac{1}{2} m c^{2}\left[1-\frac{1}{\left(1+\frac{I_{n l}}{2 m c^{2}}\right)}\right]$ and $f=\eta I_{r} /\{(l+1)(1+\xi Z)\}$, here $\eta=1.77$ and $\xi=.018$ are the experimentally fitted parameter [23].

In this paper we have $\mathrm{c}=$ velocity of light, $\mathrm{E}=$ Projectile energy, $\mathrm{m}=$ rest mass of electron, $b_{n l}=$ Bethe parameter of $n l$ subshell, $s b_{n l}=\frac{4 \pi a_{0}^{2} R^{2} N_{i}}{I_{r}^{2}}$, R=Rydberg energy, $N_{i}=$ number of electrons in $i^{\text {th }}$ subshell, $a_{0}=$ first Bohr radius, $I_{n l}=$ ionization thresholds energy of $n l$ subshell. $l=$ angular quantum number, $\beta=\mathrm{v} / \mathrm{c}, \mathrm{v}=$ velocity of the incident particle.

Bethe parameters $b_{n l}[24]$ are $.353, .265$ and .288 for $L_{1}, L_{2}$ and $L_{3}$ sub shells respectively. Since incident positron is distinguishable from the atomic electrons, the exchange scattering does not occur in positron-atom collision. So for positron, we have taken exchange factor $F_{e x}=1$ and maximum energy lost by projectile $W_{\max }=\mathrm{E}^{\prime}$ and integrated the Eq. (9) of Ref.[17]. We get Mott cross section for positron impact

$$
\sigma_{i P M}=\left(\frac{s}{t^{\prime}+f}\right)\left[1-\frac{1}{t^{\prime}}+\frac{1}{2 t^{\prime 2}}\right]
$$


where $t^{\prime}=\frac{E^{\prime}}{I_{r}}$ We have followed Khare et al. [13] to obtain $\mathrm{E}^{\prime}$ for the ionization of different subshells of an atoms

$$
E^{\prime}=E_{r}-\frac{h I}{1+F_{n l}(x)}
$$

$h=\frac{4}{3}$ for, $L_{1}(2 \mathrm{~s})$ subshell $h=\frac{8}{5}$ for $L_{2}\left(2 p_{\frac{1}{2}}\right)$ and $L_{3}\left(2 p_{\frac{3}{2}}\right)$

$$
\begin{gathered}
F_{L 1}(x)=\frac{x^{3}\left(8-5 x+x^{2}\right)}{48+48 x+24 x^{2}+6 x^{4}} \\
F_{L 1}(x)=\frac{x^{5}}{120+120 x+60 x^{2}+20 x^{3}+5 x^{4}} \quad \text { where } \mathrm{i}=2 \& 3
\end{gathered}
$$

Khare et al. [17] have taken exchange factor $F_{e x}=1$ to obtain Bethe cross section so, for positron, this term will be unchanged. In this term we have replaced $t$ by $t^{\prime}=E^{\prime} / I_{r}$. The cross sections due to transverse interaction have been taken same for both electron and positron.

\section{Result and discussion}

In present investigation the $L_{1}, L_{2}$ and $L_{3}$ sub shells ionization cross sections have been calculated for gold atom by modify the Khare [BEB] model. The ionization potentials are taken from Deslaux [25].

Figs. 1-6 the products of the present $L_{1}, L_{2}$ and $L_{3}$ sub shells ionization cross sections $\sigma L_{i}$ and the square of corresponding threshold energies $I_{n l}$, due to electron and positron impact, for gold atom are shown as a function of the over voltage $U(E / I)$. In such a representation $I^{2} \sigma_{L i}$ are expected to be independent of atomic number $\mathrm{Z}$ [1].

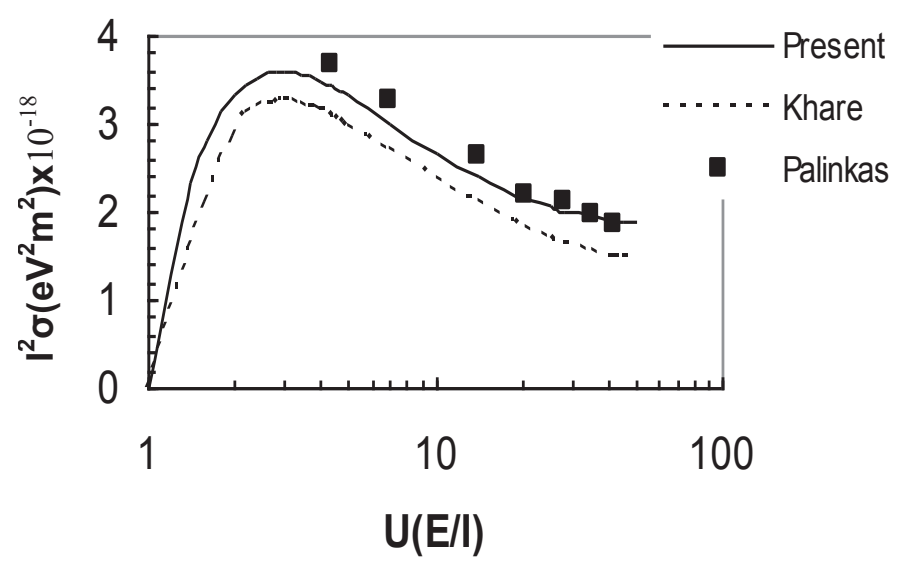

Figure 1: The present theoretical electron impact ionization cross section for L1 subshell of gold shown by line $(-)$ compared with experimental result given by Palinkas shown by square $(\boldsymbol{\square})$ and theoretical results of the Khare depicts by dash line (- - ). 
Fig. 1 shows the comparison of the present $L_{1}$ sub shell ionization cross sections by electron impact along with the experimental data given by Palinkas et al. [3] and theoretical results of the Khare et al. [13]. The ionization cross sections are in good agreement with experiment data within $8 \%$. However the calculated cross sections by Khare et al. [13] underestimate the experimental data.

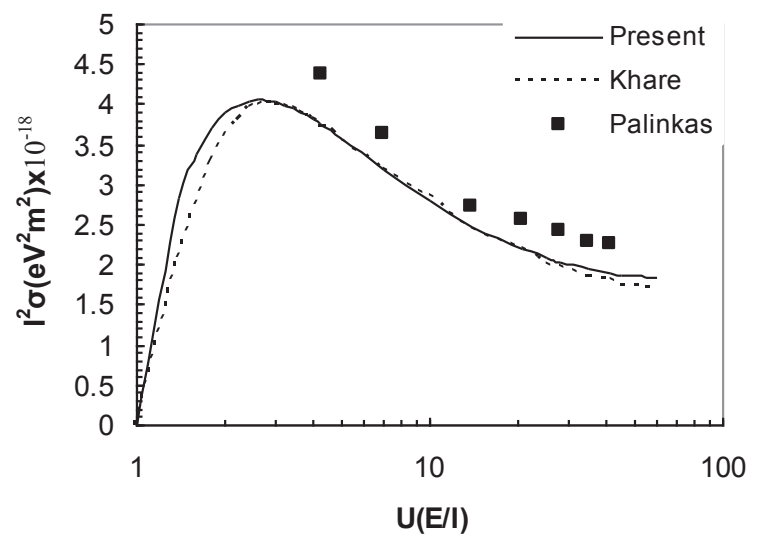

Figure 2: The present theoretical electron impact ionization cross section for L2 subshell of gold depicts by line $(-)$ compared with experimental result given by Palinkas depicts by square $(\boldsymbol{\square})$ and theoretical result of the Khare depicts by dash line (- - ).

Fig. 2, $L_{2}$ sub shell ionization cross sections, for electron impact, are shown. The present cross sections agree well with the experimental data measured by Palinkas et al. [3]. At low overvoltage our cross sections are slightly greater than the theoretical results calculated by Khare et al. [13].

Fig. 3, the present $L_{3}$ sub shell ionization cross section is compared with the exper-

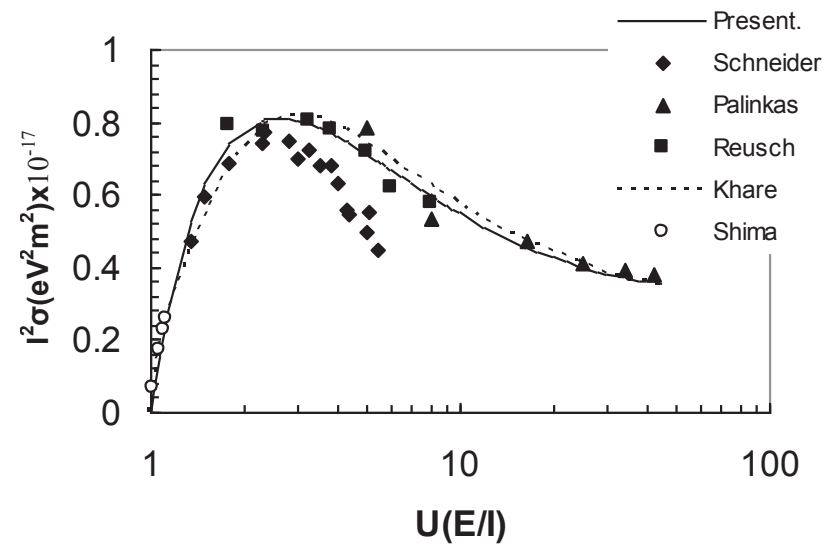

Figure 3: The present theoretical electron impact ionization cross section for L3 subshell of gold shown by solid line $(-)$ compared with the experimental results given by Schneider Palinkas, Reusch, Shima, and theoretical results of Khare shown by diamond suit $(\checkmark)$ triangle $(\mathbf{\Lambda})$ square $(\boldsymbol{\square})$ circle $(0)$ and dash line (- - -) respectively. 
imental values measured by Schneider et al. [8], Palinkas et al. [3], Reusch et al. [10] Shima et al. [6] and theoretical values of Khare et al. [13]. At low energies, the present calculated values are excellent agreed with experimental results of Shima et al. [6]. The present values agree within 10\% with the experimental values of Palinkas et al. [3] and Reusch et al. [10] The agreement between the present theoretical cross sections and the experimental data of Schneider et al. [8] remain quite good up to maximum of theoretical curve. However beyond the maximum the experimental data of Schneider et al. [8] fall at much faster rate than predicted by our calculations with overvoltage $U$.

Fig. 4 and $5, L_{1} \& L_{2}$ subshell cross sections for positron impact are shown with along theoretical cross sections of Khare et al. [13]. There are no experimental data to compare. However figure shows that present cross sections are slightly greater than to the theoretical results calculated by Khare et al. [13] at low overvoltage.

$L_{3}$ sub shell ionization cross sections by positron impact have been shown in the Fig. 6. The present calculated cross sections overestimate the experimental data. However

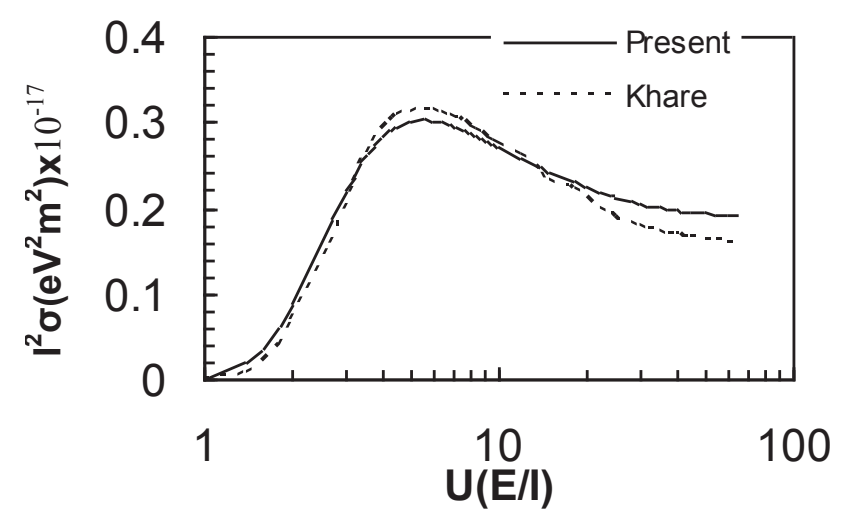

Figure 4: The present theoretical electron impact ionization cross section for L1 subshell by positron depicts by line $(-)$ compared with Khare shown by dash line (- - -).

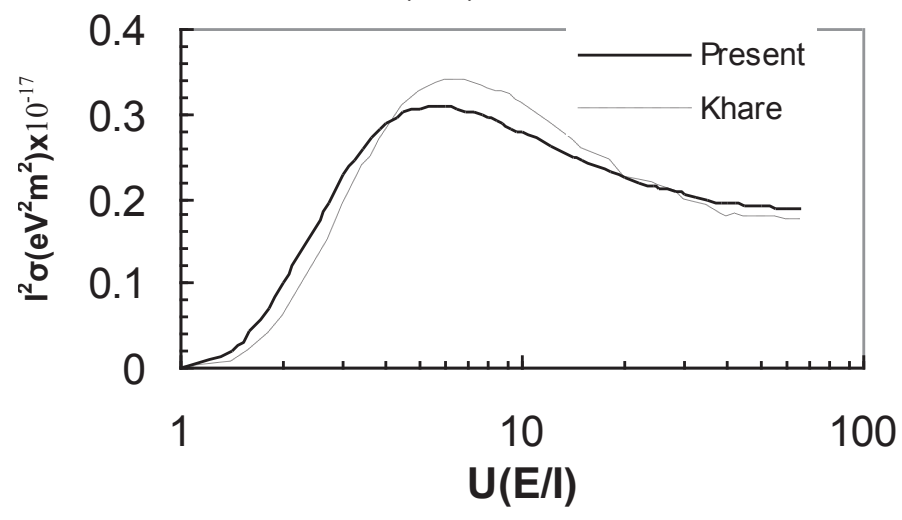

Figure 5: The present theoretical electron impact ionization cross section for L2 subshell by positron representing line (-) compared with Khare depicts by dash line (- - ). 


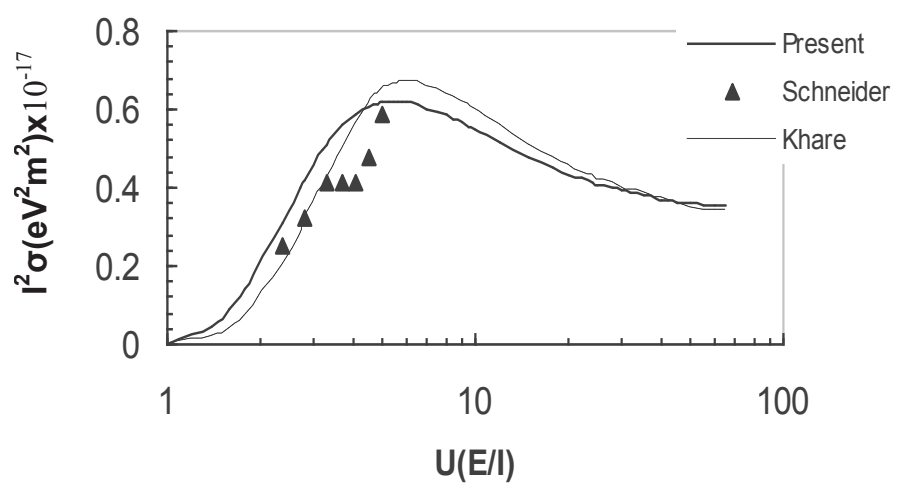

Figure 6: The present theoretical electron impact ionization cross section for L3 subshell by positron represented by line (-) compared with theoretical result calculated by Khare depicts by dash (- - -) and experimental result given by Schneider depicts by triangle $(\boldsymbol{\Lambda})$.

theoretical cross sections of Khare et al. [13] values are in good agreement with the experimental data of Schneider et al. [8].

Fig. 7, present calculated ratio of the cross sections $\sigma(e-) / \sigma(e+)$ for $L_{3}$ sub shell as a function of impact energy are compared with the experimental data of Schneider et al. [8], Lennard et al. [9] and theoretical results of Khare et al. [12]. At low impact energy the ratio obtained by Schneider et al. [8] are greater than the unity. However those obtained by Lennard et.al.[9] are less than unity over wide range of energies. The present calculated ratio $\sigma(e-) / \sigma(e+)$ are slightly lower the experimental values of Schneider $e t$ al. [8], however at high energies they are very close to each other. Schneider et al. [8] have also obtained coulomb corrected plane wave approximation (CCPWBA) cross sections. For $L_{3}$ sub shell ionization the ratio well agree with these values. However calculated

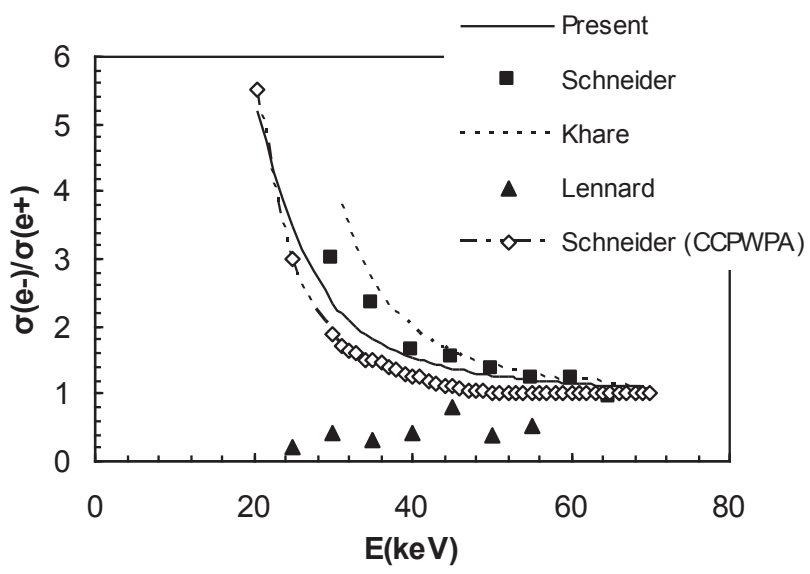

Figure 7: The present electron impact ionization cross section for L3 subshell depeits by line (-) compared with the experimental data given by Schneider depicts by square ( $\boldsymbol{\square})$ and Lennard depicts by triangle $(\boldsymbol{\Lambda})$ and theoretical results of Khare represented by dash (-- -) and also given by Schneider (CCPWBA) shown by (- -). 


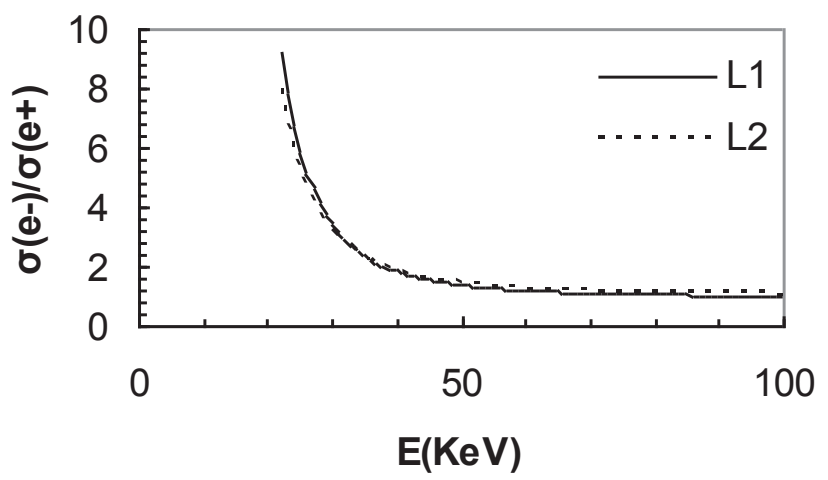

Figure 8: The ratio of cross sections and impact energy for L1 subshell represents by (-) line compared with the L2 subshell represented by dash line(- - -).

results by Khare et al. [12] do not agree with these values.

The ratio of the cross sections $\sigma(e-) / \sigma(e+)$ for $L_{1}$ and $L_{2}$ sub shells as a function of impact energy are shown in the Fig. 8. There are no experimental data to compare. As expected at low energies the ionization cross section by positron impact are less than those of electron impact for both subshells while the ratio $\sigma(e-) / \sigma(e+)$ are very close to unity in high energy region.

Fig. 9 shows the comparison of the present cross sections with the experimental data Uddin et al. [16] and the theoretical cross sections calculated by Scofield et al. [11] for L shell ionization. The present cross-sections has excellent agreement with the experimental data those measured by Palinka et al. [3] at medium energies. At high energies the calculated values agree with Middleman et al. [4] and Ishii et al. [5] within 6\%. Present

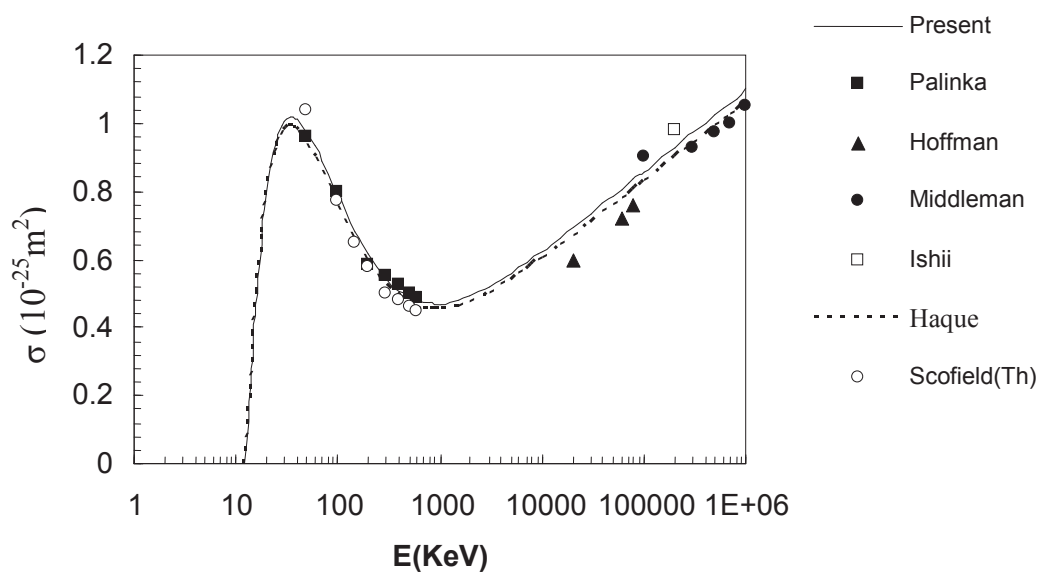

Figure 9: The present theoretical electron impact ionization cross section for $L$ shell depicts by line $(-)$ compared with the theoretical result given by Scofield depecits by circle $(0)$ and experiment result calculated by haque dash line (- - ) Palinks Square ( $\boldsymbol{\square})$ Haffman triangle $(\boldsymbol{\Delta})$ Middleman depicts by bullet $(\bullet)$ and ishii represented by white square $(\square)$. 
calculations are also in good agreement with experiment values of Hoffmann et al. [7].

\section{Conclusion}

Finally, we conclude that slight modifications in Khare (BEB) model has considerable improved the agreement between the theory and the experiment data. The available experimental data are in satisfactory agreement with the present cross section over a wide range. The application of the present model to the ionization of inner shell for other atoms is of interest.

Acknowledgments. The authors are grateful to Ex. Prof. S. P. Khare of C. C. S. University, Meerut, India for fruitful discussion.

\section{References}

[1] C. J. Powell, Electron Impact Ionization, eds. T. D. Mark and C. H. Dunn (Springer, Verlag, 1985) Ch. 6.

[2] C. E. Brion, Comm. At. Mol. Phys. 16 (1985) 249.

[3] J. Palinka and B. Schelik, Z. Phys. A 297(1980) 29.

[4] L. M. Middleman, R. L. Ford, and R. Hofstadter, Phys Rev. A 2 (1970) 1429.

[5] K. Ishii, M. Kamiya, K. Sera, S. Morita , H. Tawara, M. Oxyamada, and T. C. Chu, Phys. Rev.15 (1977) 906.

[6] K. Shima, T. Nakagawa, K. Umetani, and T. Mikumo, Phys. Rev. A 24 (1981) 72.

[7] D. H. Hoffmann, C. Brendel , H. Genz, W. Low, and S. Muller Z. Phys. A 293 (1979) 187.

[8] H. Schneinder I. Tobehn, F. Ebel, and R. Hippler Phys. Letts. A 156 (1991) 303; Hyp. Int. 73 (1992) 17.

[9] W. N. Lennard, P. J. Schultz, G. R. Mossoumi, and L. R. Logan, Phys. Rev. Lett. 61 (1988) 2428.

[10] S. Reusch, H. Genz, W. Low and A. Richter, Z. Phys. D3 (1986) 379.

[11] J. H. Scofeild, Phys. Rev. A 18 (1978) 963.

[12] S. P. Khare, V. Saksena and J. M. Wadehra, Phys. Rev. A 48 (1993) 1209.

[13] S. P. Khare, P. Sinha and J. M. Wadehra Hyp. Int. 89 (1994).

[14] S. P. Khare and J. M. Wadehra, Can. Phys. 79 (1996) 376.

[15] Y. K. Kim, J. P. Santos, and F. Parente, Phys. Rev A 62 (2000) 052710.

[16] A. K. F. Haque, M. A. Uddin, M. A. R. Patoary, A. R. Basak, M. R. Talukder, B. C. Saha and K. R. Karim Eur. Phys. J. D (2007) 203.

[17] S. P. Khare, M. K. Sharma, and S. Tomar, J. Phys. B At. Mol. Opt. Phys. 32 (1999) 3147.

[18] Y. K. Kim, M. E. Rudd, Phys. Rev A 50 (1994). 3954.

[19] V. Saksena, M. S. Kushwaha, and S. P. Khare, Physica B 233 (1997) 201.

[20] N. Tiwari, Y. Kumar, and S. Tomar, J. At. Mol. Sci. 1 (2010) 301.

[21] M. Kumar, Y. Kumar, N. Tiwari, and S. Tomar, J. At. Mol. Sci. 4 (2013) 30.

[22] Y. Kumar, N. Tiwari, M. Kumar, and S. Tomar, Ind. J. Pure App. Phys. 48 (2010) 621.

[23] Y. Kumar, N. Tiwari, M. Kumar, and S. Tomar, J. At. Mol. Sci. 3 (2012) 122.

[24] S. P. Khare, Introduction to the theory of collisions ot electrons with atoms and molecules (Klumer Academic Press, New York, 2010).

[25] J. P. Desclaux, At. Nucl. Data Tables 12 (1973) 11325. 